\title{
HRVTool - an Open-Source Matlab Toolbox for Analyzing Heart Rate Variability
}

\author{
Marcus Vollmer ${ }^{1,2}$ \\ ${ }^{1}$ Institute of Bioinformatics, University Medicine Greifswald, Germany \\ ${ }^{2}$ German Centre for Cardiovascular Research (DZHK), Greifswald, Germany
}

\begin{abstract}
Motivation: Many software tools for ECG processing are commercial. New innovative and alternative features for heart rate variability analysis (HRV) and improved methods in ECG preprocessing cannot be incorporated. Moreover, software manuals are lacking of clarity and often conceal the exact calculation methods that makes clinical interpretation difficult, and reproducibility is reduced.

Software description: HRVTool provides an opensource and intuitive user-friendly environment for the HRV analysis in Matlab. The software is available at http://marcusvollmer.github.io/HRV and supports the processing of ECG, pulsatile waveforms and RR intervals from various sources (mat and text files containing raw data, Polar, PhysioNet, Hexoskin, BIOPAC, European Data Format, ISHNE Holter Standard Format, and Machine-Independent Beat files). An integrated heart beat detector locates R peaks or pulse waves. Visual inspection, and manual adjustments of beat locations are possible and the corresponding annotation file can be saved in a standard Matlab format or as a delimited text file. HRV statistics are computed in a sliding window to evaluate the alteration over time. HRV metrics can be exported. An animation of intervals supports pattern identification. Moreover the Matlab class (HRV.m) includes functions for windowed $H R V$ computation that can be used for batch processing.
\end{abstract}

\section{Introduction}

Heart Rate Variability (HRV) analyzes the physiological phenomenon of heart beat variation over time and is used to determine autonomic activity of the heart [1]. Disorders in the regular heart rate as a result of disturbances in the electrical system of the heart are called arrhythmia. Expert cardiologists can identify such a physiological variation of the heart rate by analyzing the ECG leads (electrocardiogram) and thereby diagnose different cardiac disorders. In contrast to arrhythmia screening, HRV as a measure of neurovegetative activity is used in remedial medicine for prognostic purposes, performance objectification and risk stratification for cardiovascular events. HRV biofeedback methods are established and heart rate analysis and continuous assessment of HRV as objective features are widely used beyond the medical environment, e.g. for assessing the fitness status, physical and mental stress level.

Many diverse HRV indices emerged and are usually classified by nature of processing (time-domain methods, spectral analysis, geometric methods) and the range of application (short and long-term HRV measures), see [1-3]. Each of the methods have their specific strength and weaknesses in capturing different characteristics of the interbeat variations.

Important for valid HRV results is the quality of the ECG and the robust extraction of inter-beat intervals (IBIs or RR intervals) because many indices are not robustly defined in such a way, that wrong RR intervals do not distort the overall analysis - although there are some robust measures such as rrHRV [4]. In general also pulsatile measurements from a fingertip photoplethysmogram or through ballistocardiography are suitable to extract IBIs.

Popular free HRV software libraries includes: $\mathrm{Ku}-$ bios [5], PhysioNet Cardiovascular Signal Toolbox [6, 7], RHRV [8, 9], ARTiiFACT [10], HRVAS [11], SinusCor [12], and hrvanalysis 1

These software libraries usually consists of data import tools with automated algorithms for IBI extraction. Modules for data screening, manual adjustments, and data cleaning are integrated. HRV indices are computed, results are shown, and the exports made available. The tools differ in providing open access to the made calculations, the usage of HRV indices, the filtering of IBIs for robust measures, accepted data formats, the style of data representation, and user-friendly handling [6].

Usually, new innovative and alternative features for heart rate variability analysis and improved methods in ECG preprocessing cannot be incorporated. We also see deficits that software manuals are lacking of clarity and often conceal the exact calculation methods that makes clinical interpretation difficult, and reproducibility is reduced.

${ }^{1}$ https://github.com/Aura-healthcare/hrvanalysis 
In this paper we present an open-source and comprehensive Matlab class with transparent code for HRV analysis and an open-source intuitive user-interface for the HRV analysis in Matlab (HRVTool v1.04 updated Sep 1, 2019). The toolbox and its supporting functions for data import and processing, except of BIOPAC import functions by Jimmy Shen, are open-source licensed under MIT license and made freely available at https://github.com/MarcusVollmer/HRV, the project page http://marcusvollmer.github.io/HRV/, and Matlab Central

\section{Computational methods}

Data import and heart beat detection Supported file types are listed in Table 1 and includes several widely used formats [13] and particular manufacturer formats.

Table 1. Table of supported files

\begin{tabular}{ll}
\hline \hline & Description \\
\hline HRM & Polar files \\
MAT & $\begin{array}{l}\text { Matlab files containing waveforms or } \\
\text { RR intervals (in ms) } \\
\text { text files containing waveforms or } \\
\text { TXT }\end{array}$ \\
& $\begin{array}{l}\text { RR intervals (in ms) } \\
\text { EhysioNet files } \\
\text { (PhysioNet wfdb toolbox required) }\end{array}$ \\
WAV & Hexoskin files \\
EDF & European Data Format \\
ACQ & BIOPAC data (Source code of Jimmy Shen) \\
ISHNE & $\begin{array}{l}\text { Holter Standard Format } \\
\text { (ECG and annotation data) }\end{array}$ \\
MIB & Machine Independent Beat file \\
MIBF & (GE Marquette holter format)
\end{tabular}

For EDF, MIB, ISHNE, and ACQ particular import functions exist in the GitHub repository, that are commented and can be used for batch processing. In case of EDF files, all signals can be imported as a Matlab structure or particular signals can be selected from a drop-down list. HRVTool allows the import of existing annotation files of heart beat locations or runs a robust automated detection algorithm [14] for the identification. Recommended parameters can be chosen from a drop-down list to allow the detection in human or animal (rat) ECGs or in pulsatile signals with fast (less accurate) or normal detection speed. Manual settings can be added to qrs_settings . mat.

HRV.m - a Matlab class for HRV analysis HRV.m constitutes an extendable and transparent Matlab class with diverse functions for continuous HRV assessment. All available methods listed in Table 2 uses matrix operations as far as possible to obtain local HRV measures from long sequences of RR intervals. Missing values are supported and no additional Matlab toolboxes are required.

Batch processing The sample batch function (available on GitHub, HRV_sample_batchjob.m) shows an ex-
Table 2. Table of available methods in HRV.m

\begin{tabular}{|c|c|}
\hline Name & Description \\
\hline SDSD & $\begin{array}{l}\text { Compute standard deviation of succes- } \\
\text { sive differences }\end{array}$ \\
\hline SDNN & $\begin{array}{l}\text { Compute standard deviation of NN inter- } \\
\text { vals }\end{array}$ \\
\hline RMSSD & $\begin{array}{l}\text { Compute root mean square of successive } \\
\text { differences }\end{array}$ \\
\hline $\mathrm{pNNx}$ & $\begin{array}{l}\text { Probability of intervals greater } x \mathrm{~ms} \text { or } \\
\text { smaller }-x \mathrm{~ms}\end{array}$ \\
\hline pNN50 & $\begin{array}{l}\text { Probability of intervals greater } 50 \mathrm{~ms} \text { or } \\
\text { smaller }-50 \mathrm{~ms}\end{array}$ \\
\hline triangular_val & Compute Triangular Index and TINN \\
\hline TRI & $\begin{array}{l}\text { Compute Triangular index from the inter- } \\
\text { val histogram }\end{array}$ \\
\hline TINN & $\begin{array}{l}\text { Compute TINN, performing Triangular } \\
\text { Interpolation }\end{array}$ \\
\hline DFA & Perform Detrended Fluctuation Analysis \\
\hline $\mathrm{CD}$ & Compute the Correlation Dimension \\
\hline ApEn & Approximate Entropy \\
\hline fft_val_fun & $\begin{array}{l}\text { Spectral analysis of an IBI sequence } \\
\text { (LF,HF,ratio) }\end{array}$ \\
\hline $\mathrm{fft}_{-} \mathrm{val}$ & $\begin{array}{l}\text { Continuous spectral analysis in a win- } \\
\text { dowed approach (LF,HF,ratio) }\end{array}$ \\
\hline returnmap_val & $\begin{array}{l}\text { Results of the Poincare plot } \\
\text { (SD1,SD2,ratio) }\end{array}$ \\
\hline HR & Compute the average heart rate \\
\hline $\operatorname{rrx}$ & Compute relative $\mathrm{RR}$ intervals \\
\hline rrHRV & $\begin{array}{l}\text { Compute HRV based on relative RR in- } \\
\text { tervals }\end{array}$ \\
\hline RRfilter & $\begin{array}{l}\text { Remove artifacts from RR sequences us- } \\
\text { ing rrx }\end{array}$ \\
\hline pattern & $\begin{array}{l}\text { Recognition of patterns and regularities } \\
\text { in data }\end{array}$ \\
\hline
\end{tabular}

emplary workflow to process many recordings in a loop. It shows how HRV measures are calculated from the HRV class and stores results in an Excel spreadsheet.

\section{Software description}

To analyze HRV and for a better understanding of the cardiovascular activity, an open-source graphical user interface (GUI) has been created with Matlab 2019a. The GUI of HRVTool is hard-coded and does not make use Matlab building apps (GUIDE/App-Designer). All components can be modified without asking. The software comes with three different color schemes: dark (default), light, and user-specific. Own colors can be set in clr_user.mat.

The Interface The HRVTool GUI is a one-page screen and starts initially with sample data, such as shown in Figure 11 The menu bar (from left to right) consists of icons enabling the 'Data import', 'Saving of settings' (appear- 

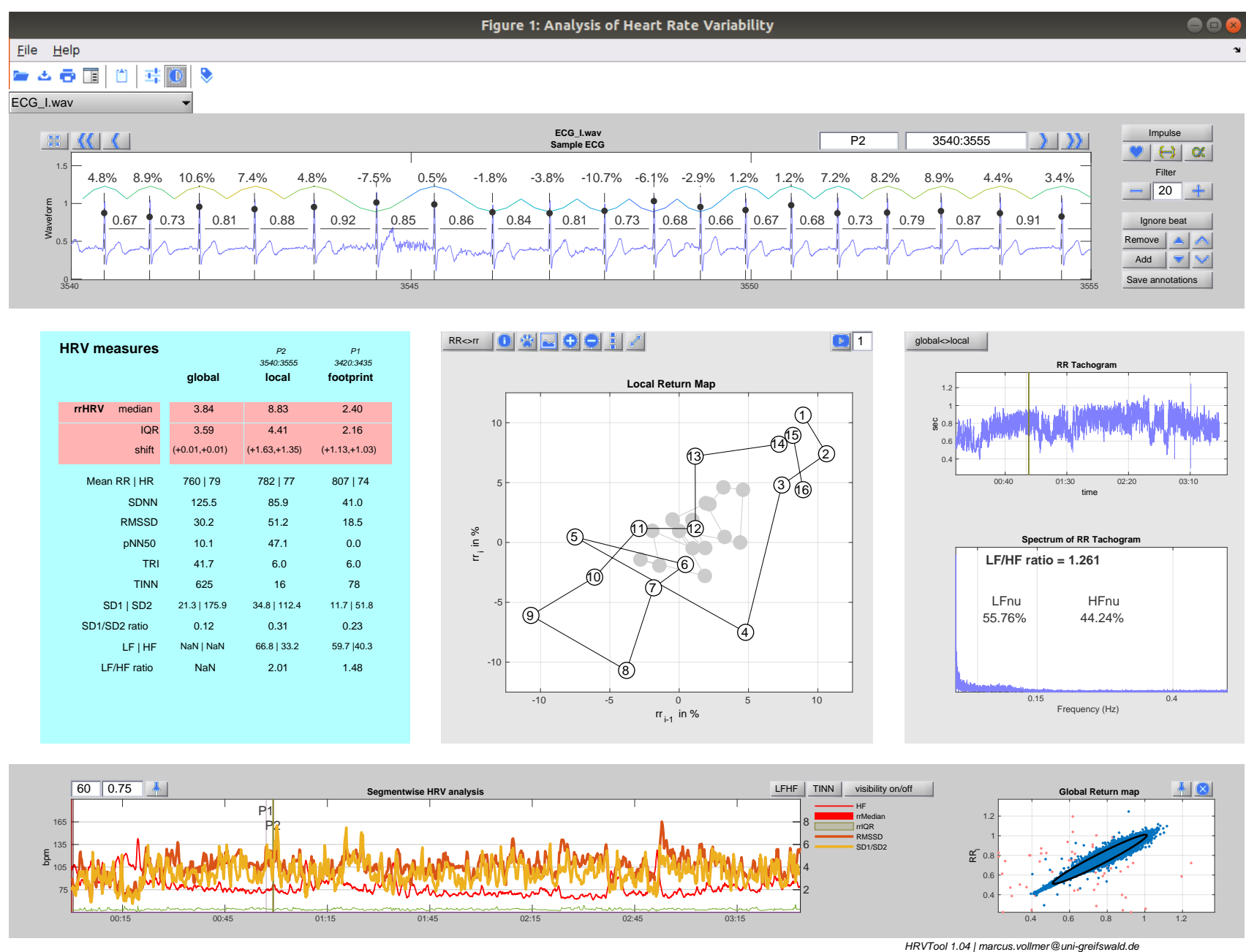

Figure 1. The graphical user interface of HRVTool with light color scheme is divided into five segments: (top) RR interval series or waveform with annotation editing, (middle-left) HRV results, (middle-center) return map of selected RR interval period with option for real time animation of interval dynamics, (middle-right) RR interval tachogram and spectral density, (bottom) time-course of heart rate and HRV parameters and return map of entire IBIs with option to mark outliers and to show the related period of RR intervals.

ance of the GUI), 'Printing', 'Matlab property inspector', 'Copy to clipboard' (of results in a Matlab matrix format), adjustment of 'Font size', selection of the 'Color scheme', and to set the 'Record title'. Furthermore, it is possible to save the analysis as a pdf figure, Matlab figure, png, mat file, or a csv file containing the HRV results as given in the screen ('files/save as').

The top module of the HRVTool shows the ECG, pulse, or IBIs in a narrowed window. Buttons can be used for navigation or the period of interest can be directly entered in the rightmost edit field above the plot, either as SS:SS or HH:MM: SS . HH:MM: SS. The selected period of data can be named by entering a name in the first edit field above the plot. The name appears in the HRV measures table (middle-left) and in the segment-wise HRV plot at the bottom. The y-axis can be scaled automatically using the leftmost button in the upper section. By default, RR Intervals or IBIs are filtered from noise, artifacts and exceptional beats using relative RR intervals [15]. The intensity can be changed (see 'Filter' manipulation right to the time series plot). Non-used IBIs are displayed as 'NaN' and not used in HRV calculations. Entering $\mathrm{NaN}$ in the filter edit field disables the filtering of RR intervals.

Current IBIs are visualized in a local return map in the middle of the screen. A button will change the display from relative to absolute pairs of IBIs. There are buttons to change the display according to the needs, and the footprint button will temporary save the results of the selected period to allow the direct comparison with other periods. The footprint is illustrated as gray dots and adds a separate column in the left handed table with HRV measures that denotes the characteristics of the footprint period. Periodic 
rhythms in the IBIs (e.g. from respiratory sinus arrhythmia) are usually visible here and complex rhythm patterns can be animated by pressing the 'play button' in the right upper corner of this module (with speed edit field). Right to the center the RR tachogram and its spectral density is plotted. The spectrum uses the fast Fourier transform on non-filtered, spline-interpolated RR interval series.

Time windowed analysis The widescreen plot on the bottom is showing HRV measures computed from a fixed number of beats in a windowed approach. A fixed number as given in the first edit field stabilizes the level of imprecision of HRV measures. The parameter in the second edit field is the degree of overlapping windows ( 0.25 means $25 \%$ overlapping, 0 means distinct periods). At each time point only past RR intervals are used and the higher impreciseness at the beginning is highlighted through a red shaded ground in the plot. The 'visibility' icon is here to hide and show certain measures. The 'pin' icon enables the selection of named periods in the experiment.

Manual correction of beat annotations HRVTool enables the editing of annotation files. Several buttons on the right of the upper plot allows the manual removal and inclusion of beat locations. Beats which should not be used in the HRV analysis can set to be 'ignored'. All or just the local beats can be aligned to the close maximum or minimum peak. Final annotation files can be saved in a free format (as mat, txt or csv files). It's recommended to visually checking outliers in pairs of successive RR intervals using the global return map in the bottom right plot by double clicking the outlier after pressing the 'pin' icon.

\section{Summary}

HRVTool constitutes an intuitive easy-to-use graphical user interface with transparent methods and convenient ways for modification or to link and edit certain methods in the HRV analysis pipeline. Ignored beats, labels of periods and GUI-related properties can be saved at any time to allow the continuation of the manual HRV analysis. For this purpose a settings file will be generated in the same directory as the input file. Moreover, batch processing is possible with the HRV class, supported by individual functions for data import and heart beat detection.

\section{References}

[1] Malik M, Bigger JT, Camm AJ, Kleiger RE, Malliani A, Moss AJ, Schwartz PJ. Heart rate variability. European Heart Journal 1996;17(3):354-381. ISSN 0195-668X.

[2] Smith AL, Owen H, Reynolds KJ. Heart rate variability indices for very short-term ( 30 beat) analysis. Part 1: survey and toolbox. Journal of clinical monitoring and computing 2013;27(5):569-576.
[3] Smith AL, Owen H, Reynolds KJ. Heart rate variability indices for very short-term (30 beat) analysis. Part 2: validation. Journal of clinical monitoring and computing 2013; 27(5):577-585.

[4] Vollmer M. A robust, simple and reliable measure of heart rate variability using relative RR intervals. In Computing in Cardiology, volume 42. ISSN 2325-8861, 2015; 609-612.

[5] Tarvainen MP, Niskanen JP, Lipponen JA, Ranta-Aho PO, Karjalainen PA. Kubios HRV - heart rate variability analysis software. Computer methods and programs in biomedicine 2014;113(1):210-220.

[6] Vest AN, Da Poian G, Li Q, Liu C, Nemati S, Shah AJ, Clifford GD. An open source benchmarked toolbox for cardiovascular waveform and interval analysis. Physiological Measurement 2018;39(10):105004.

[7] Goldberger AL, Amaral LAN, Glass L, Hausdorff JM, Ivanov PC, Mark RG, Mietus JE, Moody GB, Peng CK, Stanley HE. PhysioBank, PhysioToolkit, and PhysioNet: Components of a new research resource for complex physiologic signals. Circulation 2000;101(23):e215-e220.

[8] Rodríguez-Liñares L, Vila X, Mendez A, Lado M, Olivieri D. RHRV: an R-based software package for heart rate variability analysis of ECG recordings. In 3rd Iberian Conference in Systems and Information Technologies (CISTI 2008). 2008; 565-574.

[9] Martínez CAG, Quintana AO, Vila XA, Touriño MJL, Rodríguez-Liñares L, Presedo JMR, Penín AJM. Heart rate variability analysis with the R package RHRV. Springer, 2017.

[10] Kaufmann T, Sütterlin S, Schulz SM, Vögele C. ARTiiFACT: a tool for heart rate artifact processing and heart rate variability analysis. Behavior research methods 2011; 43(4):1161-1170.

[11] Ramshur JT. Design, evaluation, and application of heart rate variability analysis software (HRVAS). Ph.D. thesis, University of Memphis Memphis, TN, 2010.

[12] Bartels R, Neumamm L, Peçanha T, Carvalho ARS. SinusCor: an advanced tool for heart rate variability analysis. Biomedical engineering online 2017;16(1):110.

[13] Trigo JD, Alesanco Á, Martínez I, García J. A review on digital ECG formats and the relationships between them. IEEE Transactions on Information Technology in Biomedicine 2011;16(3):432-444.

[14] Vollmer M. Robust detection of heart beats using dynamic thresholds and moving windows. In Computing in Cardiology 2014. ISSN 2325-8861, Sept 2014; 569-572.

[15] Vollmer M. Arrhythmia classification in long-term data using relative RR intervals. In 2017 Computing in Cardiology (CinC), volume 44. IEEE, 2017; 1-4.

Address for correspondence:

Marcus Vollmer/marcus.vollmer@uni-greifswald.de Institute of Bioinformatics / University Medicine Greifswald

Felix-Hausdorff-Str. 8 / 17475 Greifswald / Germany 